\title{
Temperature Dependence of the Heat Capacity of Polymeric Compositions Based on Polyethylene (LDPE) with a Metal Oxide Filler
}

\author{
Abdusalam Vaxitovich Umarov ${ }^{1,}$, , Haqberdi Eshmirzayevich Khamzaev², \\ Bahodir Abdusamatovich Mirsalikhov ${ }^{1}$ \\ ${ }^{1}$ Department of Physics, Tashkent Institute of Railway Engineers, Tashkent, Uzbekistan \\ ${ }^{2}$ Department of Physics, Jizzakh State Pedagogical Institute, Tashkent, Uzbekistan
}

Email address:

abdusalom@inbox.ru (A. V. Umarov), haqberdi.hamzaev@list.ru (H. E. Khamzaev)

${ }^{*}$ Corresponding author

\section{To cite this article:}

Abdusalam Vaxitovich Umarov, Haqberdi Eshmirzayevich Khamzaev, Bahodir Abdusamatovich Mirsalikhov. Temperature Dependence of the Heat Capacity of Polymeric Compositions Based on Polyethylene (LDPE) with a Metal Oxide Filler. American Journal of Mechanical and Industrial Engineering. Vol. 5, No. 1, 2020, pp. 1-5. doi: 10.11648/j.ajmie.20200501.11

Received: September 23, 2019; Accepted: February 18, 2020; Published: March 17, 2020

\begin{abstract}
The temperature dependence of the heat capacity of polymer compositions based on polyethylene filled with copper nanoparticles was studied. Based on the analysis of the data obtained, a conclusion is made about structural rearrangements. A study of the thermal conductivity of the obtained polymer compositions with nanoscale fillers shows that various components of the filler have a significant effect on the thermal conductivity of the compositions, which is due to the ability of structure formation during their formation. Measurements of the temperature dependence of thermal conductivity and heat capacity indicate the presence of visible structural rearrangements in polymer compositions with metal oxide fillers. There are various methods in which constant temperature transitions of electrical conductivity, thermal conductivity and heat capacity are detected. e. structural restructuring of defective states of polymer compositions. It was found that the transition temperature depends on the degree of filling and crystallinity of the samples.
\end{abstract}

Keywords: Polymeric Compositions, Temperature Dependence, Heat Capacity, Thermal Conductivity, Increasing Filler, Structural Features

\section{Introduction}

It should be noted that the studies of thermophysical properties while filling with different $\%$ of filler $(\mathrm{Cu}, \mathrm{CdS})$ show that in the presence of $\mathrm{Cu}$ a smaller and homogeneous spherulite structure is formed than in the initial PE, indicating that $\mathrm{Cu}$ particles are effective nuclei of structure formation. In the presence of $\mathrm{CdS}$, an uneven, defective structure is formed by needle-shaped spherulites [1].

When changing the temperature-time conditions of sample formation, the nature of the influence of $\mathrm{Cu}$ on the polymer structure remains, while the CdS oxides change. When the melt temperature and holding time decrease, CdS particles act as typical artificial structures, which results in the formation of a uniformly small-scale LDPE structure [1, 2, 5]. With increasing temperature or duration, the formation of a large, heterogeneous and defective structure is observed. The degree of ordering of polymer compositions with metal oxide fillers is reduced. Thus, the effect of metal oxides on the structure of the polymer composition may be different depending on the technology, i.e. temperature and time modes of preparation of compositions $[1,2]$.

Change of properties depending on temperature is a very important characteristic of polymeric materials. Knowledge of temperature transitions is important, first of all, because it allows you to set the temperature range in which the polymer has sufficient mechanical strength and can be used for practical purposes as a structural material [3, 4, 7-15]. The basic concepts that characterize the thermal characteristics of polymeric materials are heat resistant, temperature resistant etc. The heat resistance of polymers is the temperature limit, which persists the necessary strength of the polymer. It is 
characterized by a temperature range in which the polymer in the form of a product retains the necessary mechanical strength and performance. Quantitative characteristic of heat resistance is the temperature at which under constant load deformation of the sample does not exceed a certain threshold [1, 2, 11-15].

For solid polymers (glassy or crystalline), heat resistance is the ability not to soften with increasing temperature, for elastomers - the ability to maintain highly elastic properties and strength with increasing temperature. The quantitative characteristic is the coefficient of heat resistance, i.e. the ratio of any mechanical property (tensile strength, elongation at break, etc.) at elevated temperatures to the corresponding values at normal temperature $[1,3,5,11-15]$.

There are a number of standardized methods to determine the heat resistance, which differ in the shape and size of test specimens, type of deformation, rate of temperature increase etc. $[1-4,7-9,11]$ So, when determining the heat resistance according to Martens cantilevered sample subjected to bending moment and record the temperature at which a certain deformation is achieved. The heat resistance of the Vic is determined by pressing the cylinder end into the sample, while fixing the temperature at which the depth of the indentation will be a certain value. The heat resistance characteristics obtained in these methods fix the softening of the polymer material only under certain accepted loading and heating regimes $[1,2,11-15]$. Under operating conditions, the heat resistance of polymers depends on both the applied load and the duration of its exposure. In this case, for glassy polymers, the heat resistance cannot exceed the glass transition temperature, and for crystalline polymers, the melting temperature.

Most of the experimental data on the specific heat of polymers refers to the temperature range, the lower limit of which corresponds to the temperature of liquid hydrogen $(\sim 20 \mathrm{~K})$, and the upper limit — the melting temperature. This temperature range is sufficient to calculate the basic thermodynamic parameters of polymers (enthalpy, entropy), which are of great technical importance, from the measured values of the specific heat capacity. Meanwhile, to find out the mechanism of the heat capacity of polymers, the most important measurements carried out at lower temperatures. The measurement of the heat capacity of polymers in the temperature range from 1 to $20 \mathrm{~K}$ is of the greatest interest for comparison of experimental data with theoretical calculations, as well as for elucidation of those features of polymers that distinguish them from low-molecular solids. Attempts to extrapolate the specific heat capacity of polymers measured at $20 \mathrm{~K}$ to the region of lower temperatures, as a rule, do not lead to meaningful results.

In the area of phase transitions (melting, crystallization), a sharp change in the heat capacity of polymers is also observed. These processes are usually studied by adiabatic calorimetry (the accuracy of which as a result of the use of electronic circuits is quite high) in a wide temperature range. On temperature dependences of heat capacity of polymers characteristic peaks which with increase in speed of heating are shifted towards the raised temperatures (thus their height increases) are shown. Such character of change of thermophysical properties at transition of polyvinyl acetate from a solid state in liquid is caused by the relaxation nature of process of softening and is connected with thermal prehistory of samples. Since the glass transition temperature of PVA is $35^{\circ} \mathrm{C}$, holding it at room temperature is equivalent to a good annealing.

Measuring the heat capacity of polymers in a wide temperature range provides information about the nature of the thermal mobility of repeating elements of the macromolecule chain and its change during phase (melting, crystallization, polymorphic transformation of the crystal) or physical (glass transition) transitions. In the low temperature region, in which most precision measurements are made, the experimental values of the heat capacity of polymers in the solid state, like the heat capacity of other solids, are a monotonically increasing function of temperature, reaching a relative "saturation" at some characteristic, the so-called Debye temperature, corresponding to the excitation of all intramolecular ("skeletal") vibrational degrees of freedom of the polymer chain. The absolute heat capacities of polymers in this temperature range (near $300 \mathrm{~K}$ ) change relatively little in the homological series, but show a noticeable dependence on the mass of repeating chain links, which can be qualitatively taken into account by the following empirical relations [1, 4, 5, 11-15].

Attempts to calculate the heat capacity of polymers on the basis of the chemical structure of the repeating link have been made repeatedly. The temperature dependence of the heat capacity of polymers has a certain specificity. It is very important that the heat capacity of amorphous and crystalline polymers differ significantly. The heat capacity of amorphous polymers is generally higher than that of partially crystalline (especially strongly crystallized) polymers. It would be interesting to find out how the heat capacity changes when the degree of crystallinity of the same polymer changes. However, only polyethylene such as a crystalline polymer can be sufficiently studied for analysis.

The values (smoothed) of the specific heat capacity of polymers as a function of temperature were borrowed directly from the tables given in the original works, or determined from large-scale graphs constructed from experimental data. The average error of tabulated values of specific heat does not exceed $0.5-1 \%$.

However, the results of an experimental study of the heat capacity of polymers at low temperatures [4] show that even under this condition above $5-10 \mathrm{~K}$, Debye's formula does not even qualitatively describe the temperature dependence of $\mathrm{C}_{\mathrm{v}}$. This is due to the fact that Debye's theory of heat capacity does not take into account the anisotropy of the interatomic interaction forces occurring in polymer chains. One of the first heat capacity theories that could be applied to describe the thermal properties of polymers was proposed in [5]. The most important experimental techniques used to measure the heat capacity 
of polymers are adiabatic and dynamic calorimetry. The relative error in determining the heat capacity using precision adiabatic calorimeters is in the range of $0.1-0.5 \%$. However, the disadvantages of this method are the need to use large (several tens of grams) sample masses, low (up to $1 \mathrm{deg} / \mathrm{min}$ ) speed of stepwise temperature increase, long intervals between temperature increases to achieve thermal equilibrium, etc. For these reasons, adiabatic calorimeters are not suitable for determining the heat capacity in the temperature range of structural transformations of the polymer (especially those occurring at high speed), and are most often used for low-temperature absolute measurements. These disadvantages are deprived of lowinertia dynamic calorimeters, which use a wide (from 0.05 to $50 \mathrm{deg} / \mathrm{min}$ and above) range of rates of continuous heating of polymer samples whose mass does not exceed $0.01-0.2 \mathrm{~g}$ [1]. The relative error of heat capacity measurement using dynamic calorimeters is usually an order of magnitude higher, but by careful calibration of the device on standard substances it can be reduced to 0.5 $1 \%$.[5]

It is known that in the field of thawing the molecular mobility of individual elements of the dynamic structure of polymer compositions, the change occurs on temperature coefficient of the studied characteristics - heat capacity and thermal conductivity, which is expressed as jumps on the temperature dependence curve. The heat capacity and thermal conductivity are to varying degrees sensitive to thawing of the thermal motion of structural elements.

The most sensitive to changes in the intensity of molecular motion is the heat capacity. In the studied temperature range of $273-373^{\circ} \mathrm{K}$, a linear increase in the temperature dependence is observed for all of the obtained compositions (Figure 1). In the range $100-450^{\circ} \mathrm{K}$ for polymer compositions, $\lambda$-shaped peaks are superimposed on the linear dependence path. Note that the filling of the initial compounds of polyethylene with various concentrations of metal nanoparticles, leads to an increase in electrical conductivity by $\approx 5$ orders of magnitude, and has a significant effect on the absolute values of heat capacity. In the temperature range $80 \leq \mathrm{T} \leq 300{ }^{\circ} \mathrm{K}$, the dependence can be represented by the equation:

$$
\mathrm{C}_{\mathrm{p}}=\mathrm{a}_{1} \mathrm{~T}+\mathrm{b}
$$

The parameters " $a$ " and " $b$ " for all the studied polymers are close in absolute values. The observed linearity $\mathrm{C}_{\mathrm{p}}(\mathrm{T})$ in the temperature range $173-473^{\circ} \mathrm{K}$, with the exception of 200 and $425^{\circ} \mathrm{K}$, cannot be the result of random superposition of optical and acoustic vibrational modes. Such behavior of $\mathrm{C}_{\mathrm{p}}(\mathrm{T})$ is rather a consistent pattern associated with the structural features of polymer compositions, specifically, the presence of strong interaction along the polymer chain due to internal chemical bonding and only a weak Van der Waals interaction between adjacent chains, as well as the formation of cluster compounds of metal oxide inclusions.

\section{Results and Discussion}

Given the quasi-one-dimensional structure of polymer chains and intermolecular interaction in close proximity of the nearest neighbors, the low-temperature heat capacity of polymer compositions can be described in terms of the Stockmeyer-Hecht model [1]. In accordance with this model in the temperature range of $100-200{ }^{\circ} \mathrm{K}$ :

$$
\mathrm{C}_{v, \mathrm{a}} \approx \mathrm{a}_{1} \mathrm{~T}_{1}+\mathrm{a}_{2} \mathrm{~T}^{1 / 2}
$$

At

$$
\frac{\hbar\left(\frac{4 \mathrm{~L}_{\mathrm{y}}}{\mathrm{M}}\right)^{1 / 2}}{\mathrm{~K}_{\mathrm{b}}} \prec \prec \mathrm{T} \prec \prec \frac{\hbar\left(\frac{16 \delta_{\mathrm{y}}}{\mathrm{M}}\right)^{1 / 2}}{\mathrm{~K}_{\mathrm{b}}}
$$

The proportionality coefficients $\mathrm{a}_{\mathrm{i}}$ in these equations are determined by the interaction constants; $\mathrm{L}_{\mathrm{y}}$ - force constant interactions of the nearest links for adjacent chains, $\delta$ - force constants for deformation of the valence angles of the chain, $\mathrm{M}$ - mass of the molecule.

To compare the results of the experiment with theory, it is necessary to get $\mathrm{C}_{\mathrm{v}}$ from $\mathrm{C}_{\mathrm{p}}$. We use the well-known thermodynamic relation $[1,2]$ :

$$
\mathrm{C}_{\mathrm{p}}-\mathrm{C}_{\mathrm{v}}=\mathrm{TV} \beta_{\mathrm{T}}^{2} / \mathrm{K}_{\mathrm{T}}
$$

where $\mathrm{T}$ is the temperature, $V$ is the molar volume, $\beta_{T}$ is the coefficient of volume expansion, and $K_{T}$ is isothermal compressibility, the values of which are equal to: $\mathrm{K}_{\mathrm{T}}=10^{-10} \mathrm{M}^{2} \cdot \mathrm{H}^{-1} ; \quad \beta_{\mathrm{T}}=2 \cdot 10^{-4} \mathrm{~K}^{-1}$; $\mathrm{V}=1,032 \cdot 10^{-5} \mathrm{M}^{3} ; \rho=1162 \mathrm{~K} \Gamma \cdot \mathrm{M}^{-3}$.

Since the difference usually grows with temperature, we will make an estimate at $\mathrm{T}=300^{\circ} \mathrm{K}$. The maximum possible difference for this temperature range is $\mathrm{C}_{\mathrm{p}}-\mathrm{C}_{\mathrm{v}}=1,24$ $\mathrm{J} / \mathrm{K}$. Obviously, such difference in the analysis of the results can be neglected.

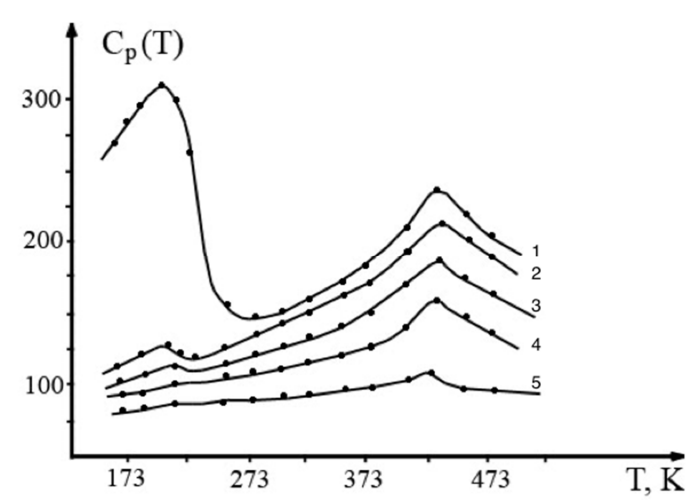

Figure 1. The temperature dependence of the heat capacity of LDPE and the LDPE + Cu composition, at: 1 - 100\% LDPE, 2 - 0.03; 3 - 0.05; 4 - 0.2; 5 $0.3 \mathrm{rpm} \mathrm{Cu}$ 
The figure 1. illustrates $\mathrm{C}_{\mathrm{p}}(\mathrm{T})$ of LDPE and compositions with $\mathrm{Cu}$. It can be seen from the figure that there is a peak on the $\mathrm{C}_{\mathrm{p}}(\mathrm{T})$ curve in the temperature range $100-225^{0} \mathrm{~K}$, which almost degenerates with increasing filler concentration. The decrease in the peak based on the degree of filling can be explained by the fact that with an increase in the degree of filling with oxides, the crystallinity of the polymer composition increases by several times, which increases the likelihood of interaction between molecular chains and metal oxide particles. If we take into account that the composite with oxide is more crystalline in comparison with the polymer, it is natural to associate the peak shift to a higher temperature region with the ordering of the structure of the polymer composition.

A study of the thermal conductivity of the obtained polymer compositions with nanoscale fillers shows that various components of the filler have a significant effect on the thermal conductivity of the compositions, which is due to the ability of structure formation during their formation.

The figure 2 shows the dependence of the coefficient of thermal conductivity on $\mathrm{V}_{1}$ compositions based on polyethylene. This shows that with an increase in the volume fraction of the filler, the thermal conductivity of the composition increases. If the most sensitive increase in thermal conductivity for compositions with $\mathrm{Cu}$ is observed at a volume of $0.1 \div 0.3$, then for compositions with $\mathrm{CdS}$ about $0.4 \div 0.5$.

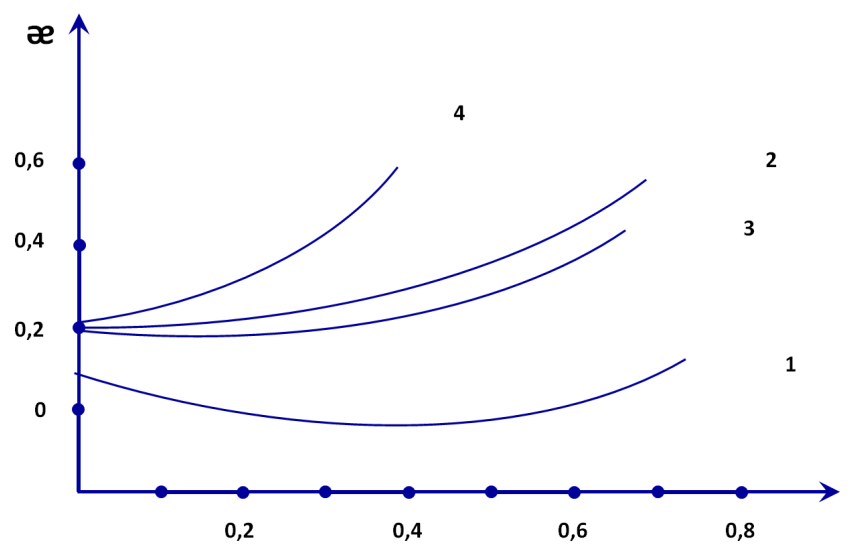

Figure 2. Concentration dependence of $P K$ thermal conductivity on the basis of PE: 1, 2 - calculations according to (5), (6) formulas.

A comparison of theoretical calculations with experimental data makes it possible to gain an idea of the structure formation of the obtained polymer compositions. According to Missner's theory for structures with cubic isolated inclusions, the thermal conductivity coefficient has the form:

$$
\chi=\chi_{2}\left(\left(1-\sqrt[3]{V_{1}}+\frac{1}{v-1}\right)+1\right)
$$

where $v=\chi_{2} / \chi_{1}, \chi_{,} \chi_{1}, \chi_{2}$ are the thermal conductivity of the composition, filler, and binder, respectively.

The Eiken-Odelevsky formula on the basis of which lies the model of an isotropic chaotic system with isomeric particles, uniformly distributed throughout the volume, for
$\mathrm{V}_{1}<\mathrm{Vcr}$

$$
\chi=\chi_{1}\left(\frac{v_{1}^{2 / 3}}{1-(1-v) V_{1}^{1 / 3}}+\left(1-V_{1}^{2 / 3}\right)\right)
$$

It can be seen from the figure that formula (6) is more suitable for our samples.

\section{Conclusion}

Thus, measurements of the temperature dependence of thermal conductivity and heat capacity revealed the presence of reversible structural rearrangements in polymer compositions with metal oxide fillers. Moreover, various methods, within error limits, detect a constant transition temperature of electrical conductivity, thermal conductivity and heat capacity, which speaks in favor of the fact that the basis of all the detected anomalies is a single mechanism, i.e. structural restructuring of defective states of polymer compositions. It was found that the transition temperature depends on the degree of filling and crystallinity of the samples.

\section{References}

[1] Lipatov Yu. S. Physicochemical fundamentals of polymer filling. M.: Chemistry, 1991.-304 p. (in Russian).

[2] Umarov A. V., Abdurakhmanov U., Development and technology of resistive composite materials, Monograph, Namangan, 2015. P. 284. (in Russian).

[3] Umarov A. B., Kasimova G. A., Askarov M. A. Investigation of the temperature dependence of the heat capacity of polymer compositions // Phys. solid, body (S.-Pb).- 1995. -37, No. 7. S. 2213-2214, (in Russian).

[4] D. Kamalova, A. Umarov, S. Negmatov, N. Abed, K. Negmatova Thermal Conductivity of SOOT Filled Compositions Based on POLYSTYRENE, Journal of Advanced Research in Science, Engineering and Technology, Vol. 5, 9, 2018, p. 6963-6968.

[5] D. P. Volkov, A. G. Egorov, M. E. Mironenko, Thermophysical properties of polimer composite materials, scientific and technical journal of information technologies, mechanics and optics, 2017, v. 17, No 2, pp. 287-293 (in Russian).

[6] D. I. Kamalova. Research of characteristics of the signal of EPR of composites. Advanced materials research. Switzerland. 2017. Vol. 1145. pp. 230-233.

[7] Mashkov Y. K., Kalistratova L. F., Kropotin O. V., The development of methods for forming effective structural phase states in polytetrafluoroethylene-based polymer composites, International polymer science and technology, 2018, Vol. 45, No 3, pp. 87-90.

[8] Sahoo NG, Cheng HKF, Cai J, Li L, Chana SH, Zhao J, Yu S. Improvement of mechanical and thermal properties of carbon nanotube composites through nanotube functionalization and processing methods. Mater Chem Phys 2009; 117: 313-320. 
[9] B. Weidenfeller, M. Hofer, F. R. Schilling, Thermal conductivity, thermal diffusivity, and specific heat capacity of particle filled polypropylene, Compos Part A Appl. Sci. Manuf. 35 (2004) 423-429.

[10] A. M. Díez-Pascual, M. Naffakh, C. Marco, G. Ellis, M. A. Gomez-Fatou, High- " performance nanocomposites based on polyetherketones, Prog. Mater Sci. 57 (2012) 1106-1190

[11] G. Makomaski, W. Ciesińska, J. Zieliński, Thermal properties of pitch-polymer compositions and derived activated carbons, Journal of Thermal Analysis and Calorimetry, 2012, Vol. 109, pp. 767-772.

[12] J. Wang, J. K. Carson, M. F. North, D. J. Cleland, A new structural model of effective thermal conductivity for heterogeneous materials with cocontinuous phases, Int. J. Heat. Mass Transf. 51 (2008) 2389-2397.

[13] Zhidong Han, Alberto Fina, Thermal Conductivity of Carbon Nanotubes and their Polymer Nanocomposites: A Review, Polymer Science, 2010, 36 (7), pp 914-944.

[14] Baronin, G., Buznik, V., Dmitriev, O. S., Zavrazhina, C., Mishchenko, S. Zavrazhin, D., Khudyakov, V.. (2017). Thermophysical properties of fluoropolymer composites with cobalt nanoparticles. AIP Conference Proceedings. 1915.
[15] Bodryakov V. Yu. Correlation of temperature dependencies of thermal expansion and heat capacity of refractory metal up to the melting point: Molybdenum / V. Yu. Bodryakov // High temperature. - 2014. - Vol. 52, iss. 6. - P. 840-845.

\section{Biography}

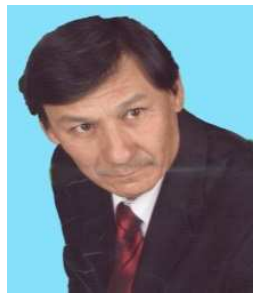

Abdusalam Vaxitovich Umarov, as one of the successors of the scientific school, he studied electrical conductivity, the influence of external factors on the electrical properties of semiconductor pyro-polymers, and also worked on the development and research of the scientific basis for creating composite thick-film resistors based on polymer or glass - with various fillers, the study of their electrical, mechanical, thermal and chemical properties and technology for creating resistors on them. Currently, he is engaged in research and development of polymer composite materials coated with nano particles. The study of their mechanical (including rheological, hardness, etc.), electrical, optical, structural and other properties. 